\title{
Krüppel-like factor 4 mediates lysophosphatidic acid-stimulated migration and proliferation of PC3M prostate cancer cells
}

\author{
Sang Hun Shin ${ }^{1,2}$, Yang Woo Kwon ${ }^{1,2}$, Soon Chul Heo ${ }^{1,2}$, Geun Ok Jeong ${ }^{1,2}$, Ba Reun Kim ${ }^{1,2}$, \\ Eun Jin Seo ${ }^{1,2}$ and Jae Ho Kim ${ }^{1,2,3,4}$
}

Prostate cancer is the most frequently diagnosed malignancy and the second leading cause of cancer mortality among men in the United States. Accumulating evidence suggests that lysophosphatidic acid (LPA) serves as an autocrine/paracrine mediator to affect initiation, progression and metastasis of prostate cancer. In the current study, we demonstrate that LPA stimulates migration and proliferation of highly metastatic human prostate cancer, PC-3M-luc-C6 cells. LPA-induced migration of PC-3Mluc-C6 cells was abrogated by pretreatment of PC-3M-luc-C6 cells with the LPA receptor 1/3 inhibitor Ki16425 or small interfering RNA (siRNA)-mediated silencing of endogenous LPA receptor 1, implicating a key role of the LPA-LPA receptor 1 signaling axis in migration of PC-3M-luc-C6 cells. In addition, LPA treatment resulted in augmented expression levels of Krüppel-like factor 4 (KLF4), and siRNA or short-hairpin RNA (shRNA)-mediated silencing of KLF4 expression resulted in the abolishment of LPA-stimulated migration and proliferation of PC-3M-luc-C6 cells. shRNA-mediated silencing of KLF4 expression resulted in the inhibition of in vivo growth of PC-3M-luc-C6 cells in a xenograft transplantation animal model. Taken together, these results suggest a key role of LPA-induced KLF4 expression in cell migration and proliferation of prostate cancer cells in vitro and in vivo.

Experimental \& Molecular Medicine (2014) 46, e104; doi:10.1038/emm.2014.34; published online 4 July 2014

Keywords: Krüppel-like factor 4; lysophosphatidic acid; migration; proliferation; prostate cancer

\section{INTRODUCTION}

Prostate cancer continues to be the most common lethal malignancy diagnosed in men and the second leading cause of male cancer deaths. ${ }^{1}$ Prostate cancer cells initially proliferate within a local area but later may metastasize, preferentially to bone, where the metastases may cause clinical problems, such as bone pain, suppressed mobility, replacement of hematopoietic tissue and compression of the spinal cord. ${ }^{2}$ Cellular motility of highly metastatic prostate cancer cells is a critical step in progression and metastasis of prostate cancer. Nevertheless, the intracellular signaling pathways essential for migration of prostate cancer cells remain poorly understood.

Lysophosphatidic acid (LPA) is increasingly being recognized as an important multifunctional mediator that affects various cellular responses, including cell proliferation, differentiation, adhesion and migration. ${ }^{3}$ LPA has been detected in many physiological and pathological biological fluids and tissues, including serum and malignant ascites from ovarian cancer patients, ${ }^{4,5}$ suggesting that LPA may serve as an autocrine/paracrine mediator in regulation of the functions of prostate cancer cells. LPA has been reported to promote the migration of prostate cancer cells, including primary prostate cancer from patients and various prostate cancer cell lines, such as LNCaP, DU145 and PC3 cells. ${ }^{6-8}$ In addition, expression of $\mathrm{LPA}$ receptors, such as $\mathrm{LPA}_{1}, \mathrm{LPA}_{2}$ and $\mathrm{LPA}_{3}$, has been detected in prostate cancer cells. ${ }^{9,10}$ In particular, $\mathrm{LPA}_{1}$ was found to have a key role in LPA-induced migration of prostate cancer cells, including LNCaP and PC3 cells. However, the downstream signaling mechanisms that mediate $\mathrm{LPA}_{1}$-activated cell migration of prostate cancer cells are still elusive.

\footnotetext{
${ }^{1}$ Medical Research Center for Ischemic Tissue Regeneration, School of Medicine, Pusan National University, Yangsan, Republic of Korea; ${ }^{2}$ Department of Physiology, School of Medicine, Pusan National University, Yangsan, Republic of Korea; ${ }^{3}$ Medical Research Institute, Pusan National University, Yangsan, Republic of Korea and ${ }^{4}$ Research Institute of Convergence Biomedical Science and Technology, Pusan National University Yangsan Hospital, Yangsan, Republic of Korea

Correspondence: Professor JH Kim, Department of Physiology, School of Medicine, Pusan National University, Yangsan, Gyeongsangnam-do 626-870, Republic of Korea. 
The Krüppel-like factor (KLF) family proteins are transcription factors implicated in the regulation of a wide range of cellular processes, including proliferation, apoptosis, differentiation, inflammation, migration and tumor formation. ${ }^{11}$ Krüppel-like factor 4 (KLF4), a member of the KLF family, is a zinc-finger transcription factor engaged in the regulation of differentiation and proliferation. ${ }^{12}$ KLF4 has recently been reported as one of four factors (Oct4, Sox2, KLF4 and c-myc) having an ability to induce reprogramming of somatic cells into an embryonic stem cell-like state or pluripotent stem cells. ${ }^{13,14}$ Expression of KLF4 in primary breast ductal carcinoma and oral squamous carcinoma in association with an aggressive phenotype has been reported. ${ }^{11}$ However, downregulation of KLF4 in several types of cancer, including prostate cancer, may contribute to cellular hyperproliferation and malignant transformation. ${ }^{15,16}$ Therefore, the role of KLF4 in tumorigenesis of prostate cancer is still unclear.

An increasing body of evidence suggests that KLF4 has a key role in chemotactic migration of several cell types, including mesenchymal stem cells, smooth muscle cells and cancer cells: ${ }^{17-19}$ oxidized phospholipids have been reported to induce migration of vascular smooth muscle through a KLF4-dependent mechanism. ${ }^{17}$ We have previously demonstrated that platelet-activating factor or an oxidized phosphatidylcholine POVPC (1-palmitoyl-2-oxovaleroyl-snglycero-3-phosphorylcholine) stimulated migration of human bone marrow-derived mesenchymal stem cells by inducing expression levels of KLF4. ${ }^{18,19}$ On the contrary, overexpression of KLF4 resulted in suppressed migration of several cell types, including vascular smooth muscle cells, MDA-MB-231 breast cancer cells and RKO human colon cancer cells. ${ }^{20-22}$ Therefore, the role of KLF4 in the regulation of migration of prostate cancer cells is still unclear.

In this study, we provide evidence of migration and proliferation of prostate cancer cells stimulated by LPA through a KLF4-dependent mechanism in vitro, as well as a key role for LPA-stimulated expression of KLF4 in tumor growth of prostate cancer in a xenograft animal model.

\section{MATERIALS AND METHODS}

\section{Materials}

RPMI 1640 medium was purchased from HyClone (Thermo Fisher Scientific, Salt Lake City, UT, USA). Phosphate-buffered saline (PBS), trypsin and Lipofectamine reagent were purchased from Invitrogen (Carlsbad, CA, USA). LPA (1-oleoyl-2-hydroxy-sn-glycero-3-phosphate) and Ki16425 were purchased from Sigma-Aldrich (St Louis, MO, USA). Platelet-derived growth factor-BB (PDGF-BB) was purchased from R\&D Systems (Minneapolis, MN, USA). Anti-KLF4 rabbit antibody (ab26648) was purchased from Abcam (Cambridge, MA, USA). Antibody against glyceraldehyde-3-phosphate dehydrogenase (GAPDH, clone MAB374) was purchased from Millipore (Billerica, MA, USA). Anti-Ki67 rabbit antibody (NCL-Ki-67p) was purchased Novocastra (Leica Microsystems, Buffalo Grove, IL, USA).

\section{Cell culture}

Luciferase-expressing PC-3M-luc-C6 cell line derived from PC-3M metastatic prostate cancer cells was purchased from PerkinElmer
(Waltham, MA, USA). PC-3M-luc-C6 cells were cultured in RPMI 1640 medium with $10 \%$ fetal bovine serum and penicillin/streptomycin, and maintained at $37^{\circ} \mathrm{C}$ in $5 \% \mathrm{CO}_{2}$. Cells were serum-starved for $24 \mathrm{~h}$ before treatment with LPA.

\section{Transfection with small interfering RNA (siRNA)}

siRNA duplexes were synthesized, desalted and purified by Samchully Pharm Co Ltd (Siheung, Gyeonggi, Korea) as follows: $\mathrm{LPA}_{1}$ $5^{\prime}$-GGACUUGGAAUCACUGUUUUU-3' (sense) and 5'-AAACAGU GAUUCCAAGUCCUU-3' (antisense); LPA $_{2} 5^{\prime}$-CCGCGAGUCUGUC CACUAUUU- $3^{\prime}$ (sense) and $5^{\prime}$-AUAGUGGACAGACUCGCGGUU- $3^{\prime}$ (antisense); LPA $_{3}$ 5'-CAGCAGGAGUUACCUUGUUUU-3' (sense) and $5^{\prime}$-AACAAGGUAACUCCUGCUGUU- $3^{\prime}$ (antisense); KLF4 $5^{\prime}$-GGTCTTGAGGAAGTGCTGA- $3^{\prime}$ (sense) and $5^{\prime}$-TGAGATGGGAA CTCTTTGTG-3' (antisense). The control siRNA (D-001206-13-05) was purchased from Dharmacon Inc. (Chicago, IL, USA).

\section{Short-hairpin RNA (shRNA)-mediated silencing of gene expression}

For generation of lentiviruses expressing shRNA, pLKO.1 constructs $(2 \mu \mathrm{g})$ were co-transfected with pVSV-G $(0.2 \mu \mathrm{g})$ and $\Delta 8.9(2 \mu \mathrm{g})$ using the calcium phosphate method in HEK293-FT cells. HEK293FT cells were cultured in Dulbecco's modified Eagle's medium with $10 \%$ fetal bovine serum and penicillin/streptomycin. Viral particles were harvested at 24 and $48 \mathrm{~h}$, and infected into cells in the presence of $8 \mu \mathrm{g} \mathrm{ml}^{-1}$ of polybrene. Puromycin $\left(10 \mu \mathrm{g} \mathrm{ml}^{-1}\right)$ was used in selection of the lentivirus-infected cells. pLKO1-puro lentiviral vectors expressing $\mathrm{LPA}_{1}$ shRNA (TRCN0000011368), KLF4 (TRCN0000005313) or non-target control shRNA (SHC002) were purchased from Sigma-Aldrich. The functional sequence in the $\mathrm{LPA}_{1}$ shRNA lentiviral vector is ' 5 '-CCGGCCTTCTGAAGACTGTGGTCAT CTCGAGATGACCACAGTCTTCAGAAGGTTTTT-3', targeting the $\mathrm{LPA}_{1}$ gene sequence (5'-CCTTCTGAAGACTGTGGTCAT-3'); the sequence of sh-KLF4 is ' 5 '-CCGGCCAGCCAGAAAGCACTACAAT CTCGAGATTGTAGTGCTTTCTGGCTGGTTTTT-3', targeting the KLF4 gene sequence (5'-CCAGCCAGAAAGCACTACAAT-3').

\section{Reverse transcription-polymerase chain reaction}

Cells were treated as indicated, and total cellular RNA was extracted using the Trizol method (Invitrogen). For reverse transcriptionpolymerase chain reaction analysis, aliquots of $2 \mu \mathrm{g}$ each of RNA were subjected to complementary DNA synthesis with $200 \mathrm{U}$ of M-MLV reverse transcriptase (Invitrogen) and $0.5 \mu \mathrm{g}$ of oligo (dT) 15 primer (Promega, Madison, WI, USA). The complementary DNA in $10 \mu \mathrm{l}$ of the reaction mixture was amplified with $0.5 \mathrm{U}$ of GoTaq DNA polymerase (Promega) and $10 \mathrm{pmol}$ each of sense and antisense

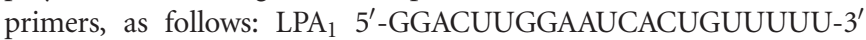
(sense), 5'-AAACAGUGAUUCCAAGUCCUU-3' (antisense), LPA 2 5'-CCGCGAGUCUGUCCACUAUUU- $3^{\prime}$ (sense), 5'-AUAGUGGACA GACUCGCGGUU- $3^{\prime}$ (antisense); LPA $35^{\prime}$-CAGCAGGAGUUACCUU GUUUU- $3^{\prime}$ (sense), $5^{\prime}$-AACAAGGUAACUCCUGCUGUU- $3^{\prime}$ (antisense); GAPDH $5^{\prime}$-TCCATGACAACTTTGGTATCG-3' (sense), $5^{\prime}$-TGTAGCCAAATTCGTTGTCA- $3^{\prime}$ (antisense). The thermal cycle profile was as follows: denaturing at $95.0^{\circ} \mathrm{C}$ for $30 \mathrm{~s}$, annealing at $52-58^{\circ} \mathrm{C}$ for $45 \mathrm{~s}$, depending on the primers used, and extension at $74.0^{\circ} \mathrm{C}$ for $45 \mathrm{~s}$. Each PCR reaction was carried out for 30 cycles, and PCR products were size-fractionated on $1.2 \%$ ethidium bromide/agarose gel and quantified under UV transillumination. 


\section{Cell migration and proliferation assays}

Cell migration assays were performed using a 96-well chemotaxis chamber (ChemoTx, Neuro Probe, Gaithersburg, MD, USA). For the migration assay, the bottom side of the ChemoTx membrane was pre-coated overnight with $20 \mu \mathrm{g} \mathrm{ml}{ }^{-1}$ rat-tail type I collagen at $4{ }^{\circ} \mathrm{C}$, and cell suspension in serum-free medium $\left(5 \times 10^{3}\right.$ cells per $\left.100 \mu \mathrm{l}\right)$ was added to the upper compartment of the chamber and separated from the lower chamber containing $100 \mu$ l of chemotactic agents by a polycarbonate filter ( $8-\mu \mathrm{m}$ pore). To induce cell migration, serumfree medium containing LPA, PDGF-BB or vehicles was added in the lower compartment of the chemotaxis system. To assess the involvement of LPA receptors in LPA-stimulated migration, the LPA receptor inhibitor Ki16425 was added into the upper compartment of the chamber. After $12-\mathrm{h}$ incubation at $37^{\circ} \mathrm{C}$ in a $5 \% \mathrm{CO}_{2}$ atmosphere, the ChemoTx membrane was fixed with $4 \%$ paraformaldehyde, and non-migratory cells on the top side of the membrane were removed by gently wiping with a cotton swab. The membrane was stained with 4,6-diamidino-2-phenylindole (DAPI) and migrating cells were counted under a fluorescence microscope at $\times 100$ magnification.

To measure the effects of LPA on cell proliferation of PC-3M-lucC6 cells, a colorimetric 3-(4,5-dimethylthiazol-2-yl)-2,5-diphenyltetrazolium bromide (MTT) assay was used: MTT is metabolized by NAD-dependent dehydrogenase to form a colored reaction product (formazan), and the amount of dye formed correlates directly with the number of cells. For determination of cell numbers, PC-3M-luc-C6 cells were seeded in a 24 -well culture plate at a density of $2 \times 10^{4}$ cells per well, cultured for $48 \mathrm{~h}$ in normal growth medium, serum-starved for $24 \mathrm{~h}$ and treated with various reagents (or a vehicle control) for the indicated times. Cells were washed twice with PBS and incubated with $100 \mu \mathrm{l}$ of MTT $\left(0.5 \mathrm{mg} \mathrm{ml}^{-1}\right)$ for $2 \mathrm{~h}$ at $37^{\circ} \mathrm{C}$. Formazan granules generated by the cells were dissolved in $100 \mu \mathrm{l}$ of dimethylsulfoxide, and the absorbance of the solution at $562 \mathrm{~nm}$ was determined using a PowerWave $_{\mathrm{x}}$ microplate spectrophotometer (Bio-Tek Instruments Inc., Winooski, VT, USA) after dilution to a linear range.

\section{Western blotting}

Serum-starved PC-3M-luc-C6 cells were treated with appropriate conditions, washed with ice-cold PBS and then lysed in lysis buffer $(20 \mathrm{~mm}$ Tris-HCl, $1 \mathrm{~mm}$ EGTA, $1 \mathrm{~mm}$ EDTA, $10 \mathrm{~mm} \mathrm{NaCl}$, $0.1 \mathrm{~mm}$ phenylmethyl sulfonyl fluoride, $1 \mathrm{~mm} \mathrm{Na} \mathrm{VO}_{4}, 30 \mathrm{~mm}$ sodium pyrophosphate, $25 \mathrm{~mm} \beta$-glycerol phosphate, $1 \%$ Triton X-100, $\mathrm{pH}$ 7.4). Lysates were resolved by sodium dodecyl sulfate-polyacrylamide gel electrophoresis, transferred onto a nitrocellulose membrane and then stained with $0.1 \%$ Ponceau S solution (Sigma-Aldrich). After blocking with $5 \%$ nonfat milk, the membranes were immunoblotted with anti-KLF4 or anti-GAPDH antibodies for $2 \mathrm{~h}$, and the bound antibodies were visualized with horseradish peroxidase-conjugated secondary antibodies using the enhanced chemiluminescence Western blotting system (GE Healthcare Life Sciences, Pittsburgh, PA, USA).

\section{Xenograft transplantation of tumor and in vivo monitoring} $\mathrm{BALB} / \mathrm{c}-\mathrm{nu} / \mathrm{nu}$ mice were randomly divided into two groups (six mice in each group). Mice in the control groups received subcutaneous injection with sh-control-infected PC-3M-luc-C6 cells $\left(1 \times 10^{6}\right.$ cells per $200 \mu \mathrm{l} \mathrm{PBS})$ and experimental groups received subcutaneous administration of sh-KLF4-infected PC-3M-luc-C6 cells $\left(1 \times 10^{6}\right.$ cells per $200 \mu \mathrm{PBS}$ ). Tumor cells were subcutaneously injected into the right and left flanks of the mice. Imaging of mice was performed before treatment and weekly during 5 weeks using a IVIS Lumina bioluminescent imager (PerkinElmer) and data analysis was performed using Xenogen living imaging software (V. 2.50). Mice were injected with D-Luciferin Firefly, potassium salt (Biosynth Inc., Itasca, IL, USA) $150 \mathrm{mg} \mathrm{kg}^{-1}$ I.P. shortly before imaging. All images were formatted with the same color-coded scale for visual assessment. The tumor volume was examined twice weekly: measurements of the length $(\mathrm{mm})$ and width $(\mathrm{mm})$ of the tumor mass were performed using electronic vernier calipers, and tumor volume $\left(\mathrm{mm}^{3}\right)$ was calculated as (length $\times$ width $\times$ width)/2. After 5 weeks, the mice were killed, and the weight of each xenograft tumor was determined.

\section{Immunofluorescence staining}

Immunostaining and confocal microscopy were used to determine the cellular expression patterns of proteins within xenograft tumor tissues. For immunostaining, specimens were incubated with antiKLF4 or anti-Ki67 antibodies for $2 \mathrm{~h}$, followed by incubation with Alexa Fluor 488-conjugated anti-rabbit secondary antibody for $1 \mathrm{~h}$. The specimens were finally washed and mounted in Vectashield medium with DAPI for visualization of nuclei. Images of KLF4positive cells, Ki67-positive cells and DAPI-positive nuclei were collected using a TCL-SP2 confocal microscope system (Leica Microsystems, Richmond Hill, Ontario, Canada).

\section{Statistics}

The results of multiple observations are presented as mean \pm s.d. Student's $t$-test was used for analysis of differences between the two groups. For multivariate data analysis, two-way analysis of variance was used for assessment of group differences, followed by post hoc comparisons tested using Scheffe's method.

\section{RESULTS}

\section{LPA induces migration of PC-3M-luc-C6 cells through an $\mathrm{LPA}_{1}$-dependent mechanism}

To explore the role of LPA in migration of prostate cancer cells, we examined the effects of LPA on chemotactic migration of PC-3M-luc-C6 cells using the ChemoTx system from Neuroprobe. LPA augmented PC-3M-luc-C6 cell migration in a concentration-dependent manner with a maximal stimulation at $1 \mu \mathrm{M}$ concentration (Figure 1a). In addition, LPA treatment resulted in stimulated migration of PC-3M-luc-C6 cells in a time-dependent manner (Figure 1b), suggesting that LPA positively regulates migration of prostate cancer cells.

To explore the role of LPA receptors in LPA-stimulated migration of PC-3M-luc-C6 cells, we examined the effects of Ki16425, an antagonist for LPA receptors 1 and 3, on the migration stimulated by LPA. As shown in Figure 2a, treatment with Ki16425 resulted in complete inhibition of LPA-stimulated migration of PC-3M-luc-C6 cells. To support the specific inhibition of LPA receptors by Ki16425, we examined the effects of Ki16425 on PDGF-BB-induced migration of PC-3M-luc-C6 cells. Treatment with Ki16425 had no significant impact on PDGF-BB-induced migration of PC-3M-luc-C6 cells, suggesting that $\mathrm{LPA}_{1 / 3}$ are responsible for LPA-induced migration of PC-3M-luc-C6 cells. Next, we examined the effects of siRNA-mediated depletion of LPA receptors on LPA-stimulated cell migration. Results of reverse transcription-polymerase chain reaction indicated expression of three LPA receptors $\left(\mathrm{LPA}_{1}, \mathrm{LPA}_{2}\right.$ and $\left.\mathrm{LPA}_{3}\right)$ in 

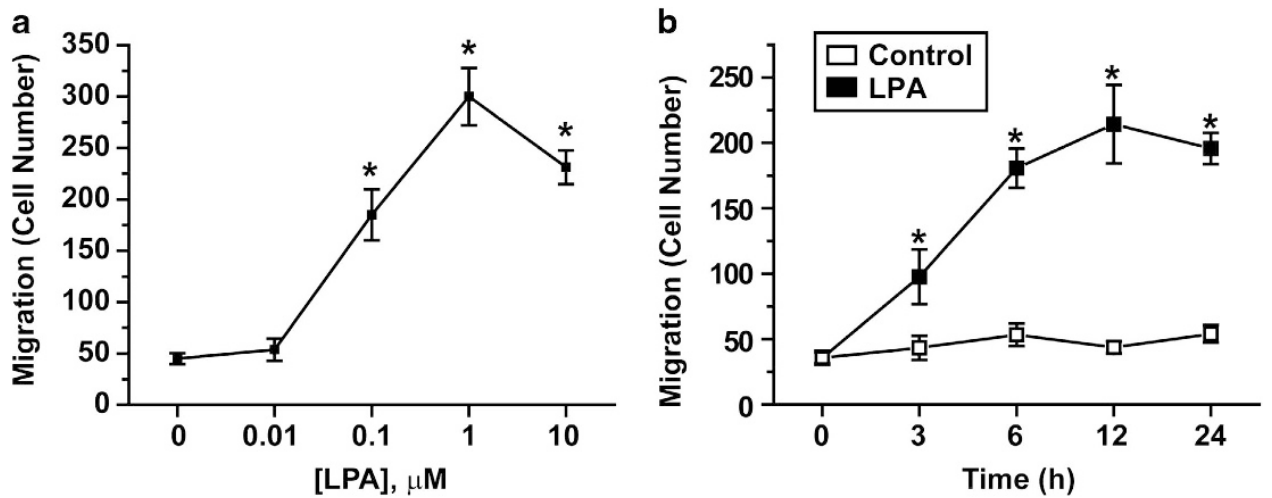

Figure 1 Effect of LPA on migration of PC-3M-luc-C6 cells. (a) Dose dependence of LPA-induced cell migration. PC-3M-luc-C6 cells were loaded into the upper compartments of a 96-well chemotaxis system and serum-free medium containing the indicated concentrations of LPA were placed in the lower chambers. The numbers of migratory PC-3M-luc-C6 cells were determined after $12 \mathrm{~h}$. (b) Time dependence of LPA-induced cell migration. Serum-free medium containing $1 \mu \mathrm{m}$ LPA or vehicles (control) were added into the lower chambers and the number of PC-3M-luc-C6 cells migrated to the lower surface of filters was determined after the indicated time periods. Data indicate mean \pm s.d. $(n=4) .{ }^{*} P<0.05$ versus control.
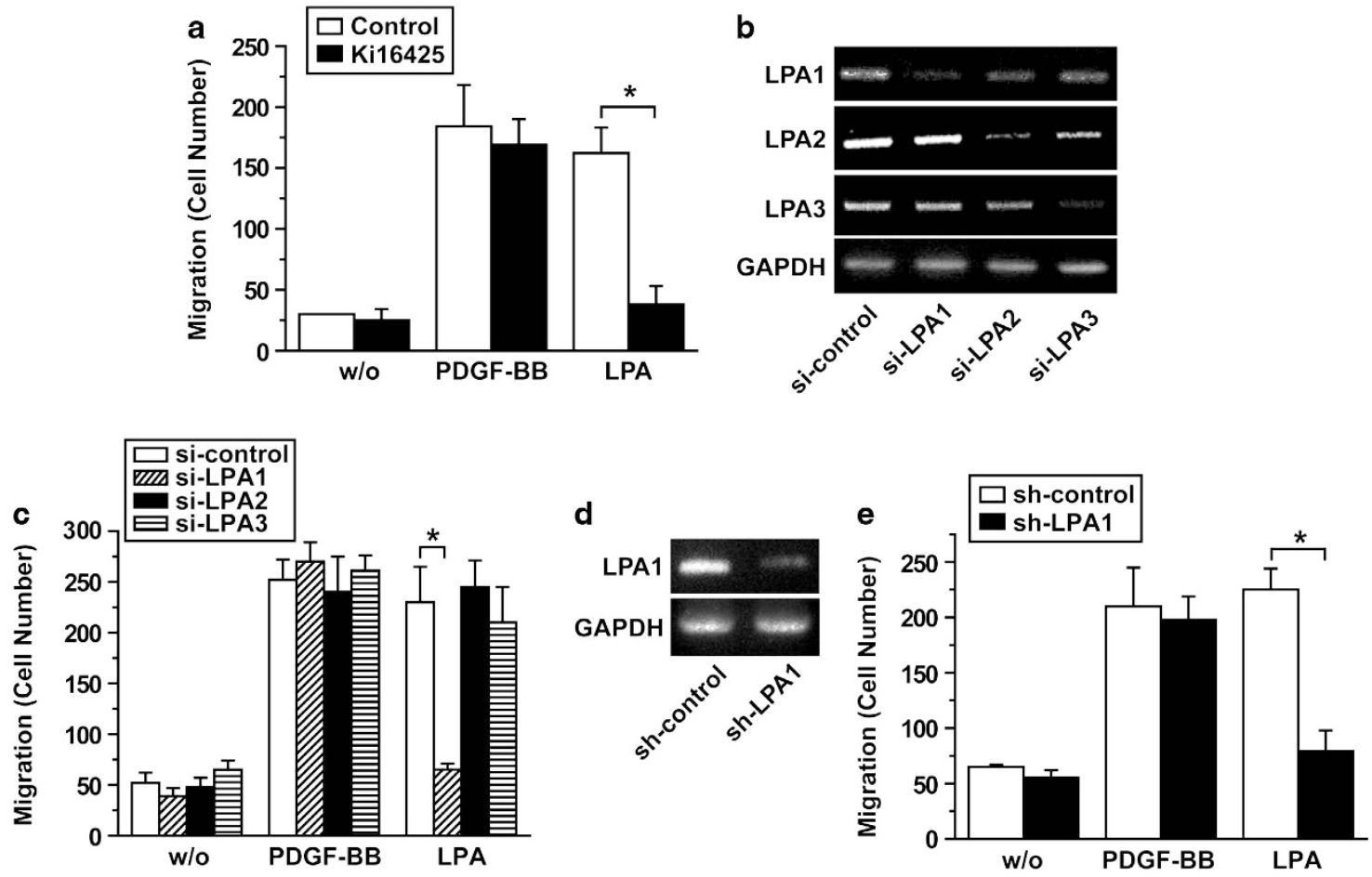

Figure 2 Role of LPA receptors in LPA-induced migration of PC-3M-luc-C6 cells. (a) Effects of Ki16425 on LPA-induced cell migration. PC-3M-luc-C6 cells were exposed to vehicles (w/o), $10 \mathrm{ng} \mathrm{ml}^{-1}$ PDGF-BB or $1 \mu \mathrm{M}$ LPA in the absence or presence of $1 \mu \mathrm{M}$ Ki16425, followed by measurement of cell migration. (b) PC-3M-luc-C6 cells were transfected with control siRNA or siRNAs Specific for LPA 1 , LPA 2 or $\mathrm{LPA}_{3}$, respectively. Reverse transcription-polymerase chain reaction (RT-PCR) analysis was performed for determination of mRNA levels of $\mathrm{LPA}_{1}, \mathrm{LPA}_{2}, \mathrm{LPA}_{3}$ and GAPDH. (c) Effects of siRNA-mediated silencing of LPA receptors on cell migration. siRNA-transfected PC-3Mluc-C6 cells were treated with vehicles (w/o), $10 \mathrm{ng} \mathrm{ml}^{-1}$ PDGF-BB or $1 \mu \mathrm{M}$ LPA for $12 \mathrm{~h}$, followed by determination of the numbers of

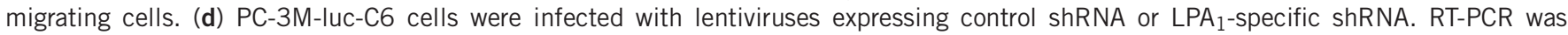
performed for determination of mRNA levels of LPA $A_{1}$ and GAPDH. (e) Effects of $\mathrm{LPA}_{1}$-specific shRNA on LPA-induced cell migration. shRNA-infected PC-3M-luc-C6 cells were treated with vehicles, $10 \mathrm{ngml} \mathrm{m}^{-1}$ PDGF and $1 \mu \mathrm{M}$ LPA, followed by determination of the numbers of migrated cells. Data indicate mean \pm s.d. $(n=4)$. ${ }^{*} P<0.05$.

PC-3M-luc-C6 cells (Figure 2b). The mRNA levels of $\mathrm{LPA}_{1}$, $\mathrm{LPA}_{2}$ or $\mathrm{LPA}_{3}$ receptors in PC-3M-luc-C6 were specifically downregulated by transfection with specific siRNAs for $\mathrm{LPA}_{1}$,
$\mathrm{LPA}_{2}$ or $\mathrm{LPA}_{3}$. Next, we examined the effects of silencing of endogenous expression of $\mathrm{LPA}_{1}, \mathrm{LPA}_{2}$ or $\mathrm{LPA}_{3}$ receptors on LPA-stimulated migration. LPA-stimulated migration of 
PC-3M-luc-C6 cells was markedly attenuated by depletion of the endogenous $\mathrm{LPA}_{1}$ (Figure 2c), whereas depletion of the endogenous expression of $\mathrm{LPA}_{2}$ and $\mathrm{LPA}_{3}$ did not affect LPAstimulated migration of PC-3M-luc-C6 cells. In addition, PDGF-BB-induced cell migration was not affected by silencing of $\mathrm{LPA}_{1}, \mathrm{LPA}_{2}$ or $\mathrm{LPA}_{3}$ receptor, respectively. To confirm these results, we explored the effect of shRNA-mediated silencing of $\mathrm{LPA}_{1}$ expression on LPA-stimulated cell migration. $\mathrm{LPA}_{1}$ expression was downregulated by lentiviral infection of $\mathrm{LPA}_{1}$-specific shRNA (Figure 2d). shRNA-mediated silencing of $\mathrm{LPA}_{1}$ expression blocked the migration of PC-3M-luc-C6 cells stimulated by LPA but not PDGF-BB (Figure 2e). These results clearly indicate that LPA has a key role in LPAstimulated migration through activation of $\mathrm{LPA}_{1}$, but not $\mathrm{LPA}_{2}$ or $\mathrm{LPA}_{3}$.

KLF4 mediates LPA-stimulated migration and proliferation of PC-3M-luc-C6 cells

To explore the question of whether LPA treatment can induce expression of KLF4, PC-3M-luc-C6 cells were treated with LPA for $6 \mathrm{~h}$ and western blot analysis was then performed for determination of the expression levels of KLF4. As shown in Figure 3a, treatment with LPA resulted in a dose-dependent increase in the expression level of KLF4, with a maximal stimulation at $1 \mu \mathrm{M}$. In addition, treatment of PC-3M-luc-C6 cells with LPA showed a time-dependent increase in expression of KLF4 with a maximal stimulation at $6 \mathrm{~h}$ (Figure $3 \mathrm{~b}$ ).

We have previously reported on KLF4-mediated migration of human bone marrow-derived mesenchymal stem cells induced by oxidized low-density lipoprotein or POVPC. ${ }^{19}$ In order to explore the question of whether KLF4 is involved in LPA-induced migration of prostate cancer cells, we examined
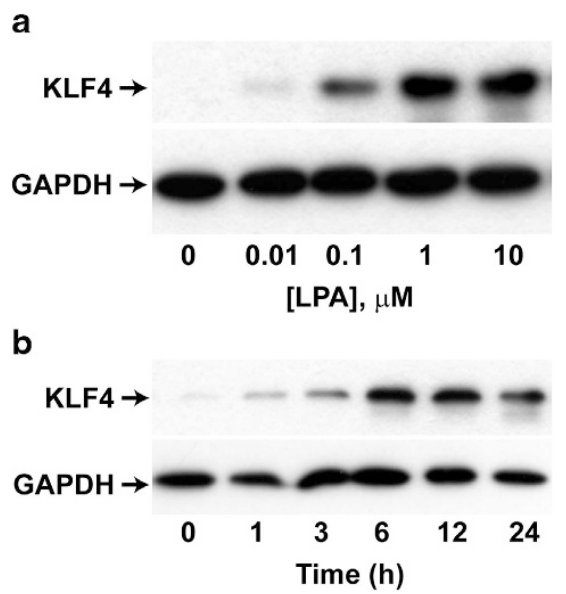

Figure 3 Effect of LPA on KLF4 expression in PC-3M-luc-C6 cells. (a) Dose dependence of LPA-induced KLF4 expression. Serumstarved $\mathrm{PC}-3 \mathrm{M}-\mathrm{luc}-\mathrm{C6}$ cells were treated with the indicated concentration of LPA for $6 \mathrm{~h}$. (b) Time dependence of LPA-induced KLF4 expression. Serum-starved PC-3M-luc-C6 cells were treated with $1 \mu \mathrm{m}$ LPA for the indicated time periods. Western blotting was performed for determination of expression levels of KLF4 and GAPDH. Representative data from three independent experiments are shown. the effects of siRNA-mediated silencing of KLF4 expression on LPA-stimulated migration of PC-3M-luc-C6 cells. Transfection of PC-3M-luc-C6 cells with KLF4-specific siRNA resulted in depleted expression of KLF4 (Figure 4a). siRNA-mediated knockdown of endogenous KLF4 in PC-3M-luc-C6 cells resulted in abrogation of LPA-induced migration of PC-3Mluc-C6 cells (Figure 4b). In order to provide support for these results, we further explored the effect of shRNA-mediated silencing of KLF4 on LPA-stimulated cell migration. As shown in Figures 4c and d, depletion of KLF4 expression with KLF4-specific shRNA showed inhibition of LPA-stimulated migration of PC-3M-luc-C6 cells. These results suggest a pivotal role of KLF4 in LPA-stimulated migration of PC-3Mluc-C6 cells.

Next, we explored the question of whether the $\mathrm{LPA}_{1}-\mathrm{KLF} 4$ signaling pathway is implicated in LPA-stimulated cell proliferation of PC-3M-luc-C6 cells. PC-3M-luc-C6 cells were infected with lentiviruses expressing control shRNA, KLF4specific shRNA or $\mathrm{LPA}_{1}$-specific shRNA, followed by treatment with LPA for 3 days, and the numbers of PC-3M-luc-C6 cells were counted. LPA treatment resulted in augmented proliferation of PC-3M-luc-C6 cells, whereas shRNA-mediated silencing of KLF4 or $\mathrm{LPA}_{1}$ in PC-3M-luc-C6 cells led to marked attenuation of LPA-induced cell proliferation (Figure $4 \mathrm{e}$ ). Taken together, these results suggest that LPA-stimulated KLF4 expression may have critical roles in cell migration and proliferation of PC-3M-luc-C6 cells.

\section{Knockdown of KLF4 expression inhibits in vivo tumor growth of PC-3M-luc-C6 cells}

To explore the role of KLF4 in tumorigenesis, we examined the effect of silencing of KLF4 in PC-3M-luc-C6 cells on tumor growth in vivo. Using shRNA, we depleted KLF4 expression, followed by subcutaneous transplantation of PC-3M-luc-C6 cells into nude mice. As shown in Figures $5 \mathrm{a}$ and $\mathrm{b}$, xenograft transplantation of control shRNA-infected PC-3M-luc-C6 cells, but not sh-KLF4-infected cells, resulted in formation of tumors. Control shRNA-infected PC-3M-luc-C6 cells, but not sh-KLF4-infected cells, exhibited a time-dependent increase in tumor volume (Figure 5c). Consistently, shRNA-mediated silencing of KLF4 resulted in reduced in vivo growth of PC3M-luc-C6 xenograft tumors (Figure 5d), suggesting a critical role of KLF4 in tumor growth of PC-3M-luc-C6 cells in vivo. In addition, in order to determine whether expression of KLF4 was knocked down by infection with KLF4-specific shRNA in vivo, we performed immunofluorescence staining using Ki67 and KLF4 in tumor tissues. Injection of PC-3M-luc-C6 cells infected with lentivirus-expressing control shRNA resulted in detection of Ki67-positive and KLF4-positive immunoreactivity in the xenograft tumor. However, injection of PC-3M-luc-C6 cells infected with sh-KLF4 lentivirus resulted in abrogated expression of KLF4 in tumor tissues. In addition, the number of Ki67-positive cells was also decreased in xenograft tumor tissues transplanted with shKLF4-infected PC-3M-luc-C6 cells compared with the control xenograft tumor tissues (Figure 6). These results suggest a 

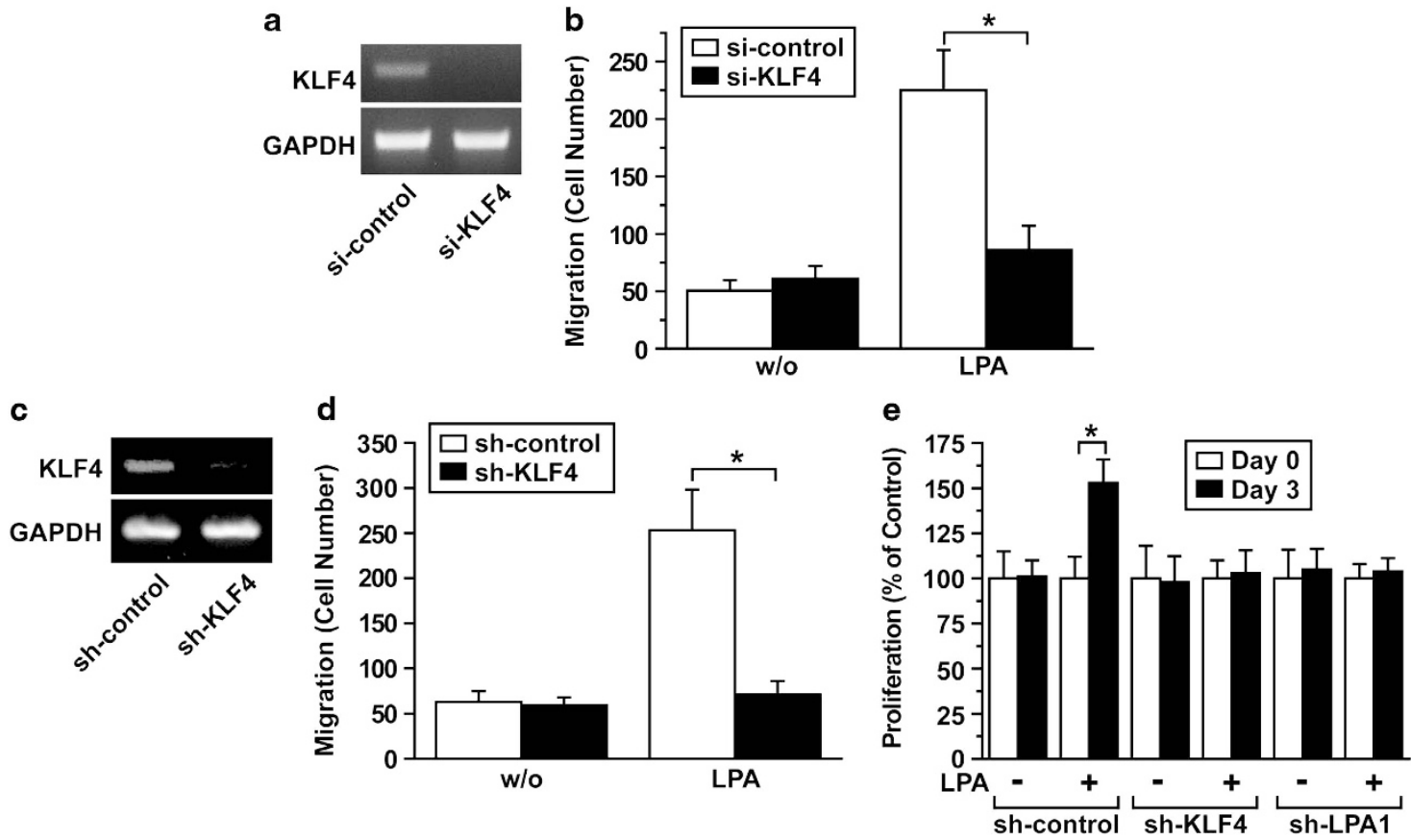

Figure 4 Role of KLF4 in LPA-induced migration and proliferation of PC-3M-luc-C6 cells. (a, c) siRNA or shRNA-mediated silencing of KLF4 expression. PC-3M-luc-C6 cells were transfected with siRNAs (a, control siRNA (si-control) or KLF4-specific siRNA (si-KLF4)) or infected with lentiviral shRNA (c, control shRNA (sh-control) or KLF4-specific shRNA (sh-KLF4)), followed by the determination of mRNA levels of KLF4 and GAPDH by RT-PCR. (b, d) Effects of KLF4 silencing on LPA-stimulated cell migration. siRNA-transfected (b) or shRNA-infected PC-3M-luc-C6 cells (d) were exposed to serum-free media containing vehicle or $1 \mu \mathrm{m}$ LPA and the number of cells that migrated to the lower surface of the filters was determined after $12 \mathrm{~h}$. (e) PC-3M-luc-C6 cells were infected with sh-control, sh-LPA ${ }_{1}$ or sh-KLF4, followed by incubation with serum-free medium containing vehicles or $1 \mu \mathrm{M}$ LPA for 3 days, and the number of cells was quantified. Data indicate mean \pm s.d. $(n=4) .{ }^{*} P<0.05$

a
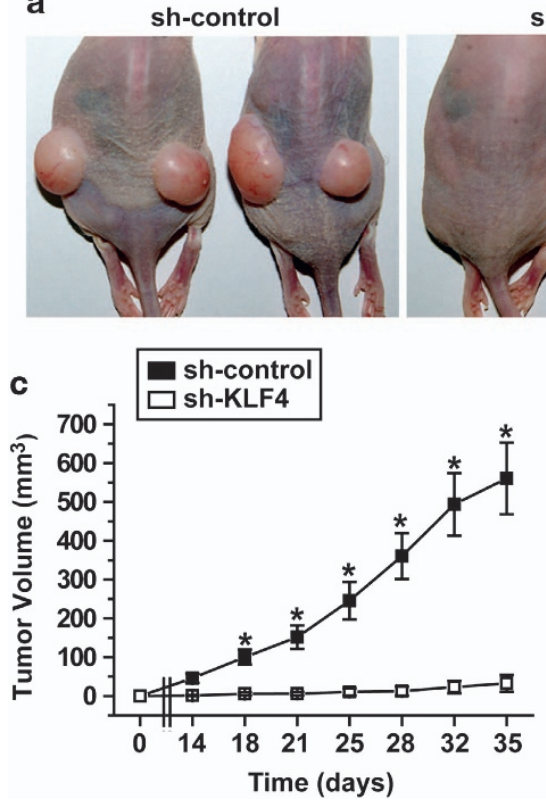

sh-KLF4

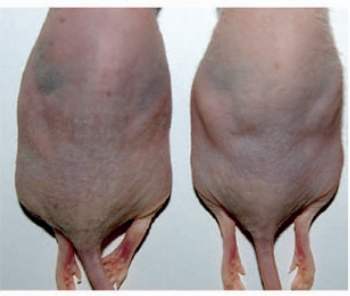

d

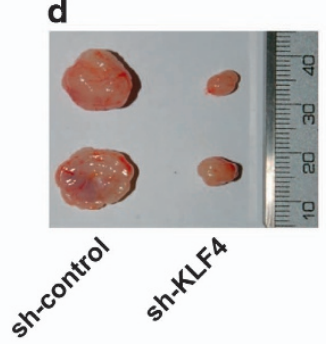

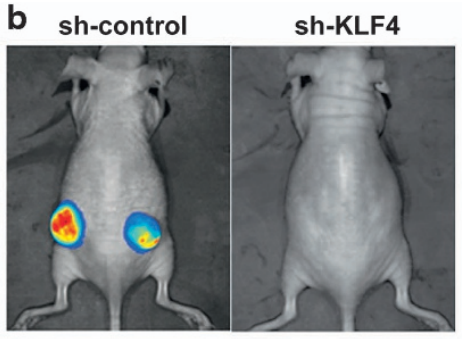

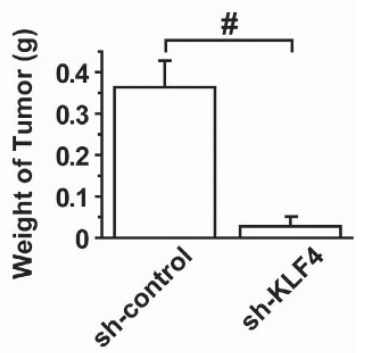

Figure 5 Role of KLF4 in growth of PC-3M-luc-C6 cells in a xenograft tumor model. (a) PC-3M-luc-C6 cells were infected with sh-control or sh-KLF4 lentiviruses and were then transplanted subcutaneously into nude mice. Mice were photographed 4 weeks after xenograft transplantation. Xenograft tumors were photographed after killing of mice. (b) Bioluminescence imaging of the xenograft tumors. (c) Volume of xenograft tumors was measured at the indicated time points. Data are expressed as mean $\pm \mathrm{s} . \mathrm{d}$. $(n=12) * P<0.05$ versus sh-control by two-way analysis of variance and Scheffe's post hoc test. (d) Weights of the tumor mass were quantified and expressed as mean \pm s.d. $(n=12)$. ${ }^{\#} P<0.05$ by Student's $t$-test. 


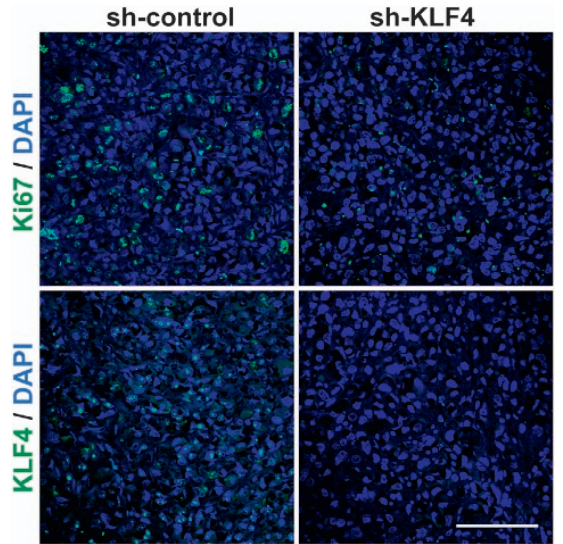

Figure 6 Role of KLF4 in proliferation of PC-3M-luc-C6 cells in the xenograft tumor. Immunostaining was performed for determination of expression of $\mathrm{Ki67}$ and KLF4 in the xenograft tissues. Images of Ki67 and KLF4 (green color) are overlaid with images of nuclei (DAPI, blue color). Scale bar $=100 \mu \mathrm{m}$.

pivotal role of KLF4 in the in vivo proliferation of human prostate tumor.

\section{DISCUSSION}

LPA has been implicated in invasion and metastasis of diverse cancer types. ${ }^{3,23}$ In the current study, we demonstrated that LPA stimulated migration of highly metastatic human prostate cancer, PC-3M-luc-C6 cells, through an $\mathrm{LPA}_{1}$-dependent mechanism. Treatment with $\mathrm{LPA}_{1 / 3}$ inhibitor Ki16425 resulted in complete abrogation of LPA-induced migration of PC-3Mluc-C6 cells. In addition, siRNA- or shRNA-mediated depletion of $\mathrm{LPA}_{1}$ showed specific abolishment of LPA-induced migration of PC-3M-luc-C6 cells. Prostate cancer cells have been shown to produce a high level of LPA and they respond to LPA via an autocrine/paracrine mechanism. ${ }^{7}$ In addition, LPA stimulated proliferation and invasion of prostate cancer cells, ${ }^{24,25}$ implying a critical role of LPA in development of prostate cancer. LPA exerts its cellular responses through its cognate $G$ proteincoupled receptors. ${ }^{26} \mathrm{LPA}_{1}$ has been implicated in LPA-induced cell migration in a variety of cell types. ${ }^{27-29}$ Together with previous reports demonstrating LPA-stimulated migration of prostate cancer cell lines through an $\mathrm{LPA}_{1}$-dependent mechanism, ${ }^{8}$ results of the current study suggest that $\mathrm{LPA}_{1}$, but not $\mathrm{LPA}_{2}$ or $\mathrm{LPA}_{3}$, is responsible for functional receptormediating LPA-stimulated migration of prostate cancer cells.

KLF4 has been implicated in differentiation during organogenesis of various tissues, including the skin, colon and eye. ${ }^{30,31}$ KLF4 also has a pivotal role in reprogramming of somatic cells for induction of pluripotent stem cells ${ }^{13}$ and maintenance of self-renewal and pluripotency of embryonic stem cells. ${ }^{32}$ Recent evidence has shown that KLF4 expression can be induced by several factors, including PDGF-BB, POVPC, platelet-activating factor and all-trans retinoic acid in vascular smooth muscle cells and human bone marrowderived mesenchymal stem cells. ${ }^{18,19,22,33,34}$ POVPC has been reported to induce migration and phenotypic switching of vascular smooth muscle cells through a KLF4-dependent mechanism. ${ }^{17}$ In the current study, we demonstrated that LPA treatment resulted in increased expression of KLF4, and siRNA- or shRNA-mediated silencing of KLF4 showed abrogation of LPA-stimulated cell migration. These results suggest a key role of KLF4 in LPA-stimulated migration of prostate cancer cells; however, further clarification of the signaling mechanisms involved in KLF4-mediated migration of PC-3M-luc-C6 cells is needed.

In this study, we demonstrated proliferation of PC-3M-lucC6 cells by LPA through a KLF4-dependent mechanism. Silencing of KLF4 expression resulted in the decrease in vivo tumor growth of transplanted prostate cancer cells. High levels of KLF4 expression were reported in primary breast ductal carcinoma and oral squamous carcinoma. ${ }^{35,36}$ It has been reported that KLF4 was required for maintenance, cell migration and invasion of breast cancer stem cells. Knockdown of KLF4 in breast cancer cells (MCF-7 and MDA-MB-231) inhibited tumorigenesis in a xenograft tumor model. ${ }^{37}$ In addition, KLF4 expression increases during progression of breast cancer and nuclear localization of KLF4 is associated with an aggressive phenotype. ${ }^{35,36}$ However, downregulated expression of KLF4 has been reported in various tumors, including colon, gastric, prostate and lung cancers, and downregulation of KLF4 expression was found to contribute to cellular hyperproliferation and malignant transformation. ${ }^{15,16}$ KLF4 expression was downregulated in hepatocellular carcinoma cells and KLF4 reverted epithelial mesenchymal transition by suppression of slug expression. ${ }^{38}$ These controversial results regarding the function of KLF4 in tumorigenesis were partially resolved by results reported from a recent study, which demonstrated that p21Cip1 status might be a switch that determines the oncogene or tumor suppressor function of KLF4. ${ }^{39}$ Therefore, these results support the current findings that KLF4 has a pivotal role in migration and proliferation of prostate cancer cells.

In conclusion, our study provides data demonstrating stimulation of cell motility of metastatic prostate cancer and tumorigenesis by LPA through a KLF4-dependent mechanism. However, further studies are needed in order to clarify the mechanisms of regulation of cell migration, proliferation and in vivo growth of prostate cancer cells by KLF4.

\section{CONFLICT OF INTEREST}

The authors declare no conflict of interest.

\section{ACKNOWLEDGEMENTS}

This study was financially supported by the 2012 Post-Doc Development program of Pusan National University.

1 Greenlee RT, Murray T, Bolden S, Wingo PA. Cancer statistics, 2000. CA Cancer J Clin 2000; 50: 7-33.

2 Nelson WG, De Marzo AM, Isaacs WB. Prostate cancer. N Engl J Med 2003; 349: 366-381.

3 Mills GB, Moolenaar WH. The emerging role of lysophosphatidic acid in cancer. Nat Rev Cancer 2003; 3: 582-591. 
4 Tigyi G, Miledi R. Lysophosphatidates bound to serum albumin activate membrane currents in Xenopus oocytes and neurite retraction in PC12 pheochromocytoma cells. J Biol Chem 1992; 267: 21360-21367.

$5 \mathrm{Xu}$ Y, Gaudette DC, Boynton JD, Frankel A, Fang XJ, Sharma A et al. Characterization of an ovarian cancer activating factor in ascites from ovarian cancer patients. Clin Cancer Res 1995; 1: 1223-1232.

6 Hao F, Tan M, Xu X, Han J, Miller DD, Tigyi G et al. Lysophosphatidic acid induces prostate cancer PC3 cell migration via activation of LPA(1), p42 and p38alpha. Biochim Biophys Acta 2007; 1771: 883-892.

7 Xie Y, Gibbs TC, Mukhin YV, Meier KE. Role for 18:1 lysophosphatidic acid as an autocrine mediator in prostate cancer cells. J Biol Chem 2002; 277: 32516-32526.

8 Guo R, Kasbohm EA, Arora P, Sample CJ, Baban B, Sud N et al. Expression and function of lysophosphatidic acid LPA1 receptor in prostate cancer cells. Endocrinology 2006; 147: 4883-4892.

9 Daaka Y. Mitogenic action of LPA in prostate. Biochim Biophys Acta 2002; 1582: 265-269.

10 Gibbs TC, Xie Y, Meier KE. Regulation of expression of EDG family receptors in human prostate cancer cell lines. Ann N Y Acad Sci 2000; 905: 290-293.

11 McConnell BB, Yang VW. Mammalian Kruppel-like factors in health and diseases. Physiol Rev 2010; 90: 1337-1381.

12 Evans PM, Liu C. Roles of Krupel-like factor 4 in normal homeostasis, cancer and stem cells. Acta Biochim Biophys Sin (Shanghai) 2008; 40: 554-564

13 Takahashi K, Yamanaka S. Induction of pluripotent stem cells from mouse embryonic and adult fibroblast cultures by defined factors. Cell 2006; 126: 663-676.

14 Yamanaka S. Strategies and new developments in the generation of patient-specific pluripotent stem cells. Cell Stem Cell 2007; 1: 39-49.

15 Choi BJ, Cho YG, Song JW, Kim CJ, Kim SY, Nam SW et al. Altered expression of the KLF4 in colorectal cancers. Pathol Res Pract 2006; 202: 585-589.

16 Wei D, Gong W, Kanai M, Schlunk C, Wang L, Yao JC et al. Drastic downregulation of Kruppel-like factor 4 expression is critical in human gastric cancer development and progression. Cancer Res 2005; 65: 2746-2754.

17 Cherepanova OA, Pidkovka NA, Sarmento OF, Yoshida T, Gan Q, Adiguzel E et al. Oxidized phospholipids induce type VIII collagen expression and vascular smooth muscle cell migration. Circ Res 2009; 104: 609-618.

18 Shin SH, Song HY, Kim MY, Do EK, Kim KH, Kim JH. Platelet-activating factor receptor mediates oxidized low density lipoprotein-induced migration of bone marrow-derived mesenchymal stem cells. Cell Physiol Biochem 2010; 26: 689-698.

19 Shin SH, Song HY, Kim MY, Do EK, Lee JS, Kim JH. Oxidized phosphatidylcholine induces migration of bone marrow-derived mesenchymal stem cells through Kruppel-like factor 4-dependent mechanism. Mo Cell Biochem 2011; 352: 109-115.

20 Dang DT, Chen X, Feng J, Torbenson M, Dang LH, Yang VW. Overexpression of Kruppel-like factor 4 in the human colon cancer cell line RKO leads to reduced tumorigenecity. Oncogene 2003; 22: 3424-3430.

21 Yori JL, Johnson E, Zhou G, Jain MK, Keri RA. Kruppel-like factor 4 inhibits epithelial-to-mesenchymal transition through regulation of E-cadherin gene expression. J Biol Chem 2010; 285: 16854-16863.

22 Wang C, Han M, Zhao XM, Wen JK. Kruppel-like factor 4 is required for the expression of vascular smooth muscle cell differentiation marker genes induced by all-trans retinoic acid. J Biochem 2008; 144: 313-321.

23 Murph M, Tanaka T, Liu S, Mills GB. Of spiders and crabs: the emergence of lysophospholipids and their metabolic pathways as targets for therapy in cancer. Clin Cancer Res 2006; 12: 6598-6602.

24 Qi C, Park JH, Gibbs TC, Shirley DW, Bradshaw CD, Ella KM et al. Lysophosphatidic acid stimulates phospholipase $D$ activity and cell proliferation in PC-3 human prostate cancer cells. J Cell Physiol 1998; 174: 261-272.
25 Kue PF, Daaka Y. Essential role for G proteins in prostate cancer cell growth and signaling. J Urol 2000; 164: 2162-2167.

26 Lin ME, Herr DR, Chun J. Lysophosphatidic acid (LPA) receptors: signaling properties and disease relevance. Prostaglandins Other Lipid Mediat 2010; 91: 130-138.

27 Hama K, Aoki J, Fukaya M, Kishi Y, Sakai T, Suzuki R et al. Lysophosphatidic acid and autotaxin stimulate cell motility of neoplastic and nonneoplastic cells through LPA1. J Biol Chem 2004; 279: 17634-17639.

28 Yamada T, Sato K, Komachi M, Malchinkhuu E, Tobo M, Kimura T et al. Lysophosphatidic acid (LPA) in malignant ascites stimulates motility of human pancreatic cancer cells through LPA1. J Biol Chem 2004; 279: 6595-6605.

29 Shida D, Kitayama J, Yamaguchi H, Okaji Y, Tsuno NH, Watanabe T et al. Lysophosphatidic acid (LPA) enhances the metastatic potential of human colon carcinoma DLD1 cells through LPA1. Cancer Res 2003; 63: $1706-1711$.

30 Segre JA, Bauer C, Fuchs E. KIf4 is a transcription factor required for establishing the barrier function of the skin. Nat Genet 1999; 22: 356-360.

31 Swamynathan SK, Katz JP, Kaestner KH, Ashery-Padan R, Crawford MA Piatigorsky J. Conditional deletion of the mouse KIf4 gene results in corneal epithelial fragility, stromal edema, and loss of conjunctival goblet cells. Mol Cell Biol 2007; 27: 182-194.

32 Jiang J, Chan YS, Loh YH, Cai J, Tong GQ, Lim CA et al. A core KIf circuitry regulates self-renewal of embryonic stem cells. Nat Cell Biol 2008; 10: 353-360.

33 Deaton RA, Gan Q, Owens GK. Sp1-dependent activation of KLF4 is required for PDGF-BB-induced phenotypic modulation of smooth muscle. Am J Physiol Heart Circ Physiol 2009; 296: H1027-H1037.

34 Pidkovka NA, Cherepanova OA, Yoshida T, Alexander MR, Deaton RA, Thomas JA et al. Oxidized phospholipids induce phenotypic switching of vascular smooth muscle cells in vivo and in vitro. Circ Res 2007; 101: 792-801.

35 Foster KW, Frost AR, McKie-Bell P, Lin CY, Engler JA, Grizzle WE et al. Increase of GKLF messenger RNA and protein expression during progression of breast cancer. Cancer Res 2000; 60: 6488-6495.

36 Pandya AY, Talley LI, Frost AR, Fitzgerald TJ, Trivedi V, Chakravarthy M et al. Nuclear localization of KLF4 is associated with an aggressive phenotype in early-stage breast cancer. Clin Cancer Res 2004; 10: 2709-2719.

37 Yu F, Li J, Chen H, Fu J, Ray S, Huang S et al. Kruppel-like factor 4 (KLF4) is required for maintenance of breast cancer stem cells and for cell migration and invasion. Oncogene 2011; 30: 2161-2172.

38 Lin ZS, Chu HC, Yen YC, Lewis BC, Chen YW. Kruppel-like factor 4, a tumor suppressor in hepatocellular carcinoma cells reverts epithelial mesenchymal transition by suppressing slug expression. PLOS ONE 2012; 7: e43593.

39 Rowland BD, Bernards R, Peeper DS. The KLF4 tumour suppressor is a transcriptional repressor of p53 that acts as a context-dependent oncogene. Nat Cell Biol 2005; 7: 1074-1082.

(c) $\odot \Theta \bigcirc$ This work is licensed under a Creative Commons
Attribution-NonCommercial-NoDerivs 3.0 Un-
ported License. The images or other third party material in
this article are included in the article's Creative Commons
license, unless indicated otherwise in the credit line; if the
material is not included under the Creative Commons license,
users will need to obtain permission from the license holder to
reproduce the material. To view a copy of this license, visit
http://creativecommons.org/licenses/by-nc-nd/3.0/

